\title{
Heliograph Imaging Based on Compressed Sensing with Mean Shift Regularization
}

\author{
Shuzhen Wang*, Ying Wang, Liya Xu, \\ Yinlong Wang, Shuyang Guan and Na Zhou \\ School of Computer Science and Technology, Xidian University, Xi'an 710071, China
}

(Received December 24, 2013; accepted March 6, 2014)

Key words: compressed sensing, heliography, mean shift, radio astronomy, synthetic aperture radar

Heliograph imaging is the process of reconstructing a solar image from sparse frequency domain data, and the compressed sensing (CS) algorithm has shown potential to accurately recover images from highly undersampled data. However, CS is sensitive to noise and often suffers from undesired convolutive artifacts. In this paper, we present an improved CS algorithm with mean shift regularization, which helps to suppress the convolutive artifacts and reconstruct the solar fine structures. A set of experiments have been conducted using both synthetic and real images. The results demonstrate that our proposed algorithm has much smaller reconstruction errors than those of other methods.

\section{Introduction}

A heliograph is a large-scale antenna array that can provide radio solar images with high-spatial, high-temporal and high-frequency resolution. The arrays directly probe the Fourier plane associated with the image tangent to the celestial sphere at the solar position and generate visibilities. Visibility coverage in the Fourier plane is incomplete as the number of antennas is finite. Consequently, recovering a solar image from measured visibilities requires solving an ill-posed deconvolution problem.

So far, various image reconstruction methods have been proposed to deal with these missing data. For example, linear methods set missing data as zero and then obtain the solar image, also called solar brightness distribution, through the inverse Fourier transform. These simple methods are generally used to acquire the initial solution for the nonlinear methods. The nonlinear methods extrapolate these missing data based on some physical facts (such as the brightness distribution of "positive") and statistical properties to obtain the "best" estimate of the solar brightness distribution. These nonlinear

*Corresponding author: e-mail: Shuzhenwang@xidian.edu.cn 
methods can be divided into two categories: the CLEAN algorithm that is based on iterative least-squares optimization and the maximum entropy method (MEM).$^{(1,2)}$ Variations and further developments of these two methods have been subsequently proposed by researchers. ${ }^{(3,4)}$ Recently, compressed sensing (CS) has achieved great success in image reconstruction. ${ }^{(5,6)}$ Wiaux et al. first introduced CS to aperture synthesis imaging.(7) Since then, several papers on radio image reconstruction using CS methods have been published. ${ }^{(8-10)}$

Despite the high effectiveness in image reconstruction, CS-based algorithms often suffer from undesired convolutive artifacts and tend to oversmooth image details. Consequently, the algorithm fails to recover the solar fine structures when used in heliograph imaging. To overcome the drawback, an improved CS-based heliograph imaging algorithm is proposed in this paper. Besides the total variation constraint, the newly designed algorithm introduces a mean shift constraint to regularize the ill-posed problem. Minimizing total variation (TV) ensures the sparsity in the gradient domain, while the mean shift constraint enforces the directional continuity in the image gradient domain. Hence, the new alogrithm shows good performance in recovering the solar fine structures when used in heliograph imaging.

Our key contributions are the introduction of the CS algorithm to heliograph imaging and the new design of an improved CS algorithm with mean shift regularization. The rest of this paper is organized as follows. Section 2 describes the basic theory of compressed-sensing-based signal reconstruction. Heliograph imaging based on CS with mean shift regularization is described in $\S 3$, and both the model and solution are studied in detail. Some simulations are described in $\S 4$, and conclusions are drawn in $\S 5$.

\section{CS-Based Signal Reconstruction}

In the CS framework, reconstruction of a signal $X \in R^{N}$ that is sparse in a base system $\boldsymbol{\Psi} \in R^{N \times N}$ requires just a small number of measured samples $Y \in R^{M}$, where $M \ll N$. This subsampling process can be represented as a projection by an $M \times N$ measurement matrix $\boldsymbol{\Phi} \in R^{M \times N}$. Therefore, the observable $Y$ can be expressed as

$$
Y=\boldsymbol{\Phi} \cdot \boldsymbol{\Psi} \cdot X=\boldsymbol{\Theta} \cdot X
$$

The newly defined matrix $\boldsymbol{\Theta}=\boldsymbol{\Phi} \cdot \boldsymbol{\Psi}$ represents an overcomplete basis.

Equation (1) expresses an underdetermined system of linear equations where the number of unknowns is larger than the number of equations. Therefore, the solution will be nonunique. To solve this equation, CS assumes that the signal is sparse, which means that the number of nonzero coefficients of $X$ in the $\Psi$-domain, $|\boldsymbol{\Psi} \cdot X|_{0}$, tends to be as small as possible.

$$
\min |\boldsymbol{\Psi} \cdot X|_{0} \text { s.t. } Y=\boldsymbol{\Theta} X
$$

Apparently, minimization of eq. (2) is a combinatorial problem that is computationally intractable. Fortunately, the solution of eq. (2) for L0 normal is identical to the solution 
of a more tractable L1 normal problem when the signal is sparse. Thus, the CS recovery of $X$ from $Y$ is formulated as the following constrained optimization problem.

$$
\min _{x}\left\|\boldsymbol{\Psi}^{T} X\right\|_{1} \text { s.t. } \boldsymbol{\Phi} \boldsymbol{\Psi}^{T} X=Y
$$

\section{Heliograph Imaging Based on CS with Mean Shift Regularization}

A heliograph does not directly measure the brightness distribution of a solar image but samples the visible complex value on the UV plane through the array, and finally obtains the solar image from the obtained data through inverse Fourier transform. Therefore, heliograph imaging can be understood as a process of the recovery of the original image $f$ from the corresponding complex visibility value $Y$ and the sampling distribution $S$ determined by the given array configuration.

\subsection{Imaging model based on CS of minimizing total variation}

Total variation is widely used to express sparseness in a $2 \mathrm{D}$ image. The minimum total variation, proposed by Candés, ${ }^{(6)}$ is suitable for $2 \mathrm{D}$ image reconstruction based on a priori knowledge that the discrete gradient of most natural images is sparse in practice. According to the CS theory, the imaging model based on CS of minimizing the total variation for heliograph is

$$
\min _{f} T V(f) \text { s.t. }\|\boldsymbol{S H} f-Y\|_{2}^{2} \leq \sigma,
$$

where $\sigma$ is a noise-related constant greater than $0, \boldsymbol{H}$ is the two-dimensional discrete Fourier transform matrix, and $\boldsymbol{S}$ is the sampling distribution. $T V(f)$ is the sum of the discrete gradient at each point of the image $f$ :

$$
\begin{gathered}
T V(f)=\sum_{i, j} \sqrt{\left(f_{\mathrm{i}+1, j}-f_{i, j}\right)^{2}+\left(f_{i, j+1}-f_{i, j}\right)^{2}}=\sum_{i, j}\left\|D_{i, j} f\right\|_{2}, \\
D_{i, j} f=\left(\begin{array}{l}
f_{i+1, j}-f_{i, j} \\
f_{i, j+1}-f_{i, j}
\end{array}\right) .
\end{gathered}
$$

\subsection{Imaging model based on CS with mean shift regularization}

The model based on CS of minimizing total variation has good performance in reconstructing extended sources but it fails to recover the solar fine structure. The reason for this is that minimizing the total variation tends to oversmooth the gradient of images which contains most of the image details. Moreover, the model often suffers from undesired convolutive artifacts because it treated the fine structure as individual pixels instead of a region. 
To overcome the drawback, mean shift regularization is introduced. Mean shift filtering is very effective in sharpening edges and preserving image details. Hence, mean shift has gained widespread popularity in the fields of clustering and segmentation.

For a given pixel $f_{i j}$ in an image $f$, its neighborhood mean, denoted by $n m\left(f_{i j}\right)$, is obtained as a weighted average of surrounding pixels within a neighborhood domain, and the mean shift of $f_{i j}$, $m s\left(f_{i j}\right)$, is given by the difference between $f_{i j}$ and $n m\left(f_{i j}\right)$, namely, $m s\left(f_{i j}\right)=f_{i j}-n m\left(f_{i j}\right)$,

$$
n m\left(f_{i j}\right)=\sum_{k=1}^{m} f_{i j} g\left(\left\|\frac{f_{i j}-f^{k}}{h}\right\|_{2}^{2}\right) / \sum_{k=1}^{m} g\left(\left\|\frac{f_{i j}-f^{k}}{h}\right\|_{2}^{2}\right),
$$

where $f^{k}$ is the pixel in the neighborhood domain, $m$ is the pixel number, and $g$ is the kernel, typically a gaussian kernel, and $h$ is the bandwidth.

Equation (6) can be expressed in vector form, $n m\left(f_{i j}\right)=W_{i j}^{T} \Lambda_{i j}$, where $\Lambda_{i j}$ is the column vector containing all the central pixels around $f_{i j}$ and $W_{i j}$ is the column vector containing all the corresponding weights $w_{i j}$. As the elements of $\Lambda_{i j}$ are all the elements of $f, m s\left(f_{i j}\right)$, together with $n m\left(f_{i j}\right)$, is the weighted sum of $f$. Let $m s\left(f_{i j}\right)=W^{i j} f$ with $W^{i j}$ be the weight matrix.

Thus, heliograph imaging based on CS with mean shift regularization can be modeled as

$$
\min _{f} \sum_{i, j}\left(\left\|D_{i, j} f\right\|_{2}+\alpha\left\|W^{i j} f\right\|_{2}\right) \text { s.t. }\|\boldsymbol{S} \boldsymbol{H} f-Y\|_{2}^{2} \leq \sigma .
$$

We introduce $\omega_{i, j}$ to approximate eq. (7) into

$$
\min _{f} \sum_{i, i}\left\|\omega_{i, j}\right\|_{2} \text { s.t. }\left\{\begin{array}{l}
\omega_{i, j}=D_{i, j} f+\alpha W^{i j} f, \forall i, j \\
\|\boldsymbol{S} \boldsymbol{H} f-Y\|_{2}^{2} \leq \sigma
\end{array} .\right.
$$

Thus, minimizing eq. (8) is equivalent to solving

$$
\min _{\omega, f}\left(\sum_{i, j}\left\|\omega_{i, j}\right\|_{2}+\frac{\beta}{2} \sum_{i, j}\left\|\omega_{i, j}-D_{i, j} f-\alpha W^{i j} f\right\|_{2}^{2}+\frac{\mu}{2}\|\boldsymbol{S H} f-Y\|_{2}^{2}\right),
$$

where $\alpha, \beta$, and $\mu$ are positive, indicating the weights of the punishment and the fidelity. Equation (9) can be solved by the alternating iterations. ${ }^{(11)}$ The main steps are as follows.

(i) Let $f$ be fixed, eq. (9) is equivalent to solving

$$
\omega^{*}=\arg \min _{\omega_{i, j}}\left(\left\|\omega_{i, j}\right\|_{2}+\frac{\beta}{2}\left\|\omega_{i, j}-D_{i, j} f-\alpha W^{i j} f\right\|_{2}\right)
$$


Then, the optimal solution to eq. (10) is

$$
\omega_{i, j}^{2}=\max \left\{\left\|D_{i, j} f+\alpha W^{i j} f\right\|-\frac{1}{\beta}, 0\right\} \frac{D_{i, j} f+\alpha W^{i j} f}{\left\|D_{i, j} f+\alpha W^{i j} f\right\|_{2}}(i=1,2, \ldots, n, j=1,2, \ldots, n)
$$

(ii) Let $\omega$ be fixed, eq. (9) becomes a quadratic function of $f$. Thus, we can obtain eq. (12) by derivation. The optimal solution to eq. (12) is apparent, which is used as the initial value of the next iteration.

$$
\left(\sum_{i, j}\left\|\left(D_{i, j}+\alpha W^{i j}\right)\right\|^{2}+\frac{\mu}{\beta}(\boldsymbol{S H})^{T}(\boldsymbol{S H})\right) f=\sum_{i, j}\left(D_{i, j}+\alpha W^{i j}\right)^{T} \omega_{i, j}+\frac{\mu}{\beta}(\boldsymbol{S H})^{T} Y
$$

(iii) Repeat steps (1) and (2). $f$ and $\omega$ alternately iterate as described above until the solution becomes convergent.

\section{Experimental Results}

Two groups of experiments are conducted to compare the performance of our approach with that of the classical Richardson-Lucy (RL) algorithm and the CS-based algorithm through the processing of the synthetic images and real images.

The fidelity of the reconstructed image is measured using relative error (Rel Err) and signal-to-noise ratio $(S N R)$. In particular, relative error is defined as $\operatorname{Rel} E r r=100 \times$ $\operatorname{norm}(u-f) / \operatorname{norm}(f)$ and $S N R$ is defined as $S N R=10 \times \lg \left(\|u\|^{2} /\|u-f\|^{2}\right)$, where $u$ is the reconstructed image and $f$ is the original image.

In addition, in our experiments, the antenna array is configured as a three-arm spiral topology and composed of 100 elements with the longest baseline of $3 \mathrm{~km}$ and the shortest baseline of $6 \mathrm{~m}$. Figures 1 and 2 show topology and sampling distribution


Fig. 1 (left). (Color online) Three-arm spiral antenna array configuration.

Fig. 2 (right). (Color online) Sampling distribution in UV plane of 3-arm spiral antenna array. 
$S$ in the UV plane, respectively, where the number of sampling points is 6917 and the sampling rate is $2.64 \%$.

The simulated solar brightness image is shown in Fig. 3(a) with a size of $512 \times$ 512 pixels in the first experiment. We set three different brightness "needlelike" point sources in the disc center for the purpose of testing the dynamic range of each algorithm. Several different brightness arc sources are used to test the shape maintenance ability of the algorithms to extended sources, and several adjacent point sources with the same brightness are used to test the resolution of the three algorithms.

The results indicate that the proposed algorithm has advantages over the RL algorithm and CS algorithm in terms of image quality, shape maintenance of the extended source, resolution of the point source and dynamic range.

The second experiment is conducted on a real solar image from the NRAO image gallery, shown in Fig. 4(a), with the size of $512 \times 512$ pixels. Figures 4(b)-4(d) give the reconstructed images employing the RL, CS and CS with mean shift regularization

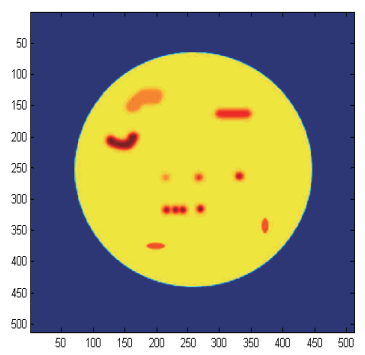

(a)



(b)



(c)



(d)

Fig. 3. (Color online) Simulated solar image and reconstructed images using 3 different algorithms. (a) Simulated solar image. (b) By RL algorithm (Rel Err $=92.3 \%, S N R=3.55)$. (c) By CS algorithm (Rel Err $=34.3 \%, S N R=32.9)$. (d) By our method (Rel Err $=22.5 \%, S N R=43.3)$.



(a)



(b)

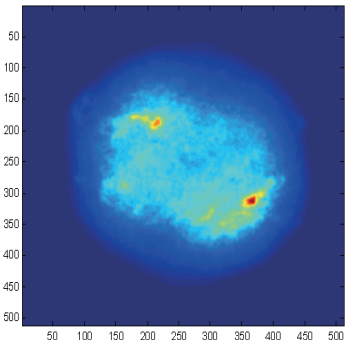

(c)



(d)

Fig. 4. (Color online) Real solar image and reconstructed images using 3 different algorithms. (a) Real solar image. (b) By RL algorithm (Rel Err $=37.5 \%, S N R=16.0)$. (c) By CS algorithm (Rel $E r r=53.1 \%, S N R=13.4)$. (d) By our method $(\operatorname{Rel} E r r=24.2 \%, S N R=32.5)$. 
algorithms, respectively. The results clearly show that the method based on CS with mean shift regularization is superior to the RL algorithm and CS algorithm in terms of image quality and dynamic range.

\section{Conclusion}

In this paper, we presented an improved CS-based image reconstruction algorithm by incorporating a mean shift regularization constraint. The total variation constraint in the CS model and mean shift regularization are complementary to each other, making the new proposed algorithm highly effective in preserving solar fine structures while eliminating the convolutive artifacts. The results of our experiments demonstrated the potential applications of the proposed method.

\section{Acknowledgements}

This work was supported by the National Natural Science Foundation of China under grant Nos. 61100156, 61070143, and 61201295.

\section{References}

1 J. A. Hogbom: Astron. Astrophys. 15 (1974) 417.

2 B. R. Frieden: J. Opt. Soc. Am. 62 (1972) 511.

3 B. Clark: Astron. Astrophys. 89 (1980) 377.

4 T. Cornwell and J. Evans: Astron. Astrophys. 143 (1985) 77.

5 Y. Tsaig and D. Donoho: Signal Process. 86 (2006) 549.

6 E. Candés, J. Romberg and T. Tao: IEEE Trans. Inf. Theory 52 (2006) 489.

7 Y. Wiaux, L. Jacques, G. Puy, A. M. M. Scaife and P. Vandergheynst: Mon. Not. R. Astron. Soc. 395 (2009) 1733.

8 S. Wenger, S. Darabi, P. Sen, K. Glassmeier and M. Magnor: Proc. Int. Conf. Image Process. (Comput. Graphics Lab., Braunschweig, 2010) pp. 1381-1384.

9 J. McEwen and Y. Wiaux: IEEE Int. Conf. Image Process. (IEEE, NJ, 2011) pp. 1313-1316.

10 J. Zhang, S. Liu, R. Xiong S. Ma and D. Zhao: 2013 IEEE Int. Symp. on Circuits and Syst. (IEEE, NJ, 2013) pp. 2836-2839.

11 Y. Wang, J. Yang, W. Yin and Y. Zhang: SIAM J. Imag. Sci. 1 (2008) 248. 